\title{
PARTICLE DYNAMICS FOR MOTION IN A NON-PLANAR ACCELERATOR
}

A. Antillon and M. Month

August 20, 1985

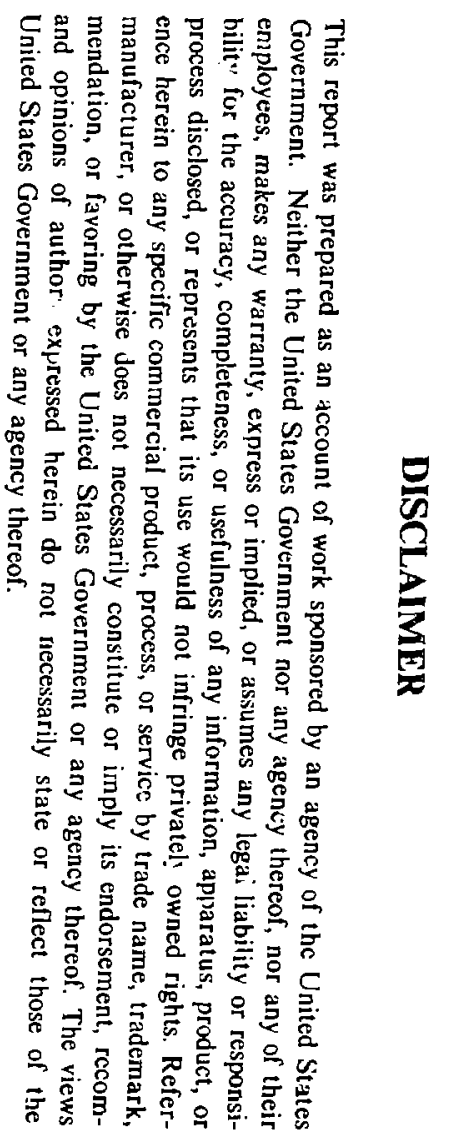

\section{ALTERNATING GRADIENT SYNCHROTRON DEPARTMENT}

BROOKHAVEN NATIONAL LÁBORATORY ASSOCIATED UNIVERSITIES. INC.

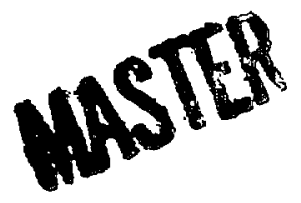
UPTON. LONG ISLAND. NEW YORK 11973 


\section{DISCLAIMER}

This report was prepared at an account of work sponsored by an agency of the linited States Government. Neither the United States Government nor any agency thereof. nor any of their employees, nor any of their contr ctors, subcontractors, or thei. employees, makes any warranty, express or implied, or assumes any legal liability or responsibility for the accuracy, completeness, ur ueefulness of any information. apparatus, product, or process disclosed, or represents that its use would not infringe privately owned rights. Reference herein to any specific commercial product. process, or service by trade name, trademark, manufart urer, or otherwise, dues not necessarily. constitute or imply its endorsement, recommendation, or favoring by the Uniter? States Government or any agency. contractor or subcontractor thereof. The views and opinions of authors expressed herein do not necessarily state or reflect those of the United States Government or any agency, contractor or subcontractor thereof

Printed in the United States of America

Available from

National Technical Information Service

U.S. Department of Commerce

5285 Port Royal Road

Springfield, VA 22161

NTIS price codes: 
Accelerator Div. Report 85-2

\title{
Particle Dynamics for Motion in a Non-Pianar Accelerator
}

\author{
A. Antillón and M. Month
}

August 20, 1985

\begin{abstract}
The basic dynamics of a planar accelerator is extended to the non-planar case. This is done using the geometrical concept of torsion and extending the Hamiltonian formalism. A generalized non-planar reference orbit is adopted which introduces torsion in appropriately chosen drift spaces. The parameters of the reference orbit are associated with uncoupled and coupled betatron parameters currently in use.
\end{abstract}

\section{DISCLAIMER}

This report was prepared as an account of work sponsored by an agency of the United States Governnient. Neither the United States Goyernment nor any agency thereof, nor any of their employees, inakes any warranty, express or implied, or assumes any legal liability or responsibility for the accuracy, completeness, or usefulness of any information, apparat"s, product, or process disclosed, or represents that its use would not infringe privately owne ights. Reference herein to any specific commercial product, process, or service by trade name, trademark, manufacturer, or otherwise does not necessarily constitute or imfly its endorsemen mendation, or favoring by the United States Government or any agency thereof. The views and opinions of authors expressed herein do not necessarily state or reflect those of the United States Government or any agency thereof. 


\section{d. Introduction}

In the development of circular accelerators, the cholce of a reference system has played an important role in the analysis of the particle dynamics. In particular, the choice has been such that a particle displaced from the reference crbit will describe, around this, a pseudoharmonic oscillation , in a coordinate system moving with the particle. Thus, implicit in our choice is the fact that the reference orbit is closed (pertodic) and that it represents a real trajectory of a particle.

Using Floquet's theorem, the periodic parameters relative to the closed reference orbit for linear magnetic systems have been obtained. However, the size of planar circulat accelerators is growing tremendously-the circumference of future accelerators will be of the order of a hundred kms. or more--and perhaps it will be desirable to follow the ground surface. This Implies that the accelerator will no longer be in a plane and planar dynamics in particular the periodic orbit parameters, wlll have to be modified. A nonplanar situation will actually exist in the operating so-called Main Ring at Fermilab in which it is planned to have an oibit which passes over the experimental facilities for colliding beams in the Tevatron. To better understand such problems, we consider here a formalism for analysis of the particle dynamics of non-planar motion and in particular show that in general such motion can be described in terms of a superimposed torsion of the reference orbit in appropriately chosen straight sections.

To accomplish this, we use a Hamiltonian formalism. We recognize that the geometrical parameter, torsion, allows us in general to extend the Courant-Snyder formalism to non-planar curves in $\mathrm{R}^{3}$, but as we will see, the reference orbit for this type of ac zelerator can be taken to be a curve in which the torsion is particularly simple. In fact, we derine the reference curve to be such that it is perpendicular to the field of each dipole and the dipole entrance so that, inside the dipoles the torsion is zero. That is, we restrict ourselves to cases where the torsion is non-zero only between dipoles. Thus, the motion within each dipole is planar. Also, we can arbitrarily select a reference orbit with no torsion in the quadrupoles. Thus, we can choose, withuut loss of generality, the case in which the torsion inside any element is zero and only has a value different from zero in drift spaces.

In Chapter 2 we obtain the general Hamfltonian for the non-planar case. In Chapter 3 we study the motion of a particle moving in a transverse field. In Section 3.1 we determine the vector potential in the introduced coordinate system, and in Section 3.2 we obtain the equations of motion around non-planar orbits. In Chapter 4, the transfer matrix for a generalized drift space (i.e. one with torsion) is analyzed. We will see that the transfer matric:ss with respect to the coordinate sets $\left(x, x^{\prime}, y, y^{\prime}\right)$ and $\left(x, P_{x}, y, P_{y}\right)$ only depend on the total angle of rotation of the coordinate frame and not on the torsion (i.e. the rate of 
coordinate spin rotation). However, the connection between these coordinate sets is dependent explicitly on the torsion. This has the implication that the physics as observed in regions of non-zero torsion is dependent on the torsion, $\tau$. To avold confusion in this situation, we have taken $\tau \neq 0$ only in the straight sections and using the fact that the transfer matrix depends only on the rotation angle and not on $\tau$ explicttly. It is interesting that a solenoid magnet introduces similar effects, specifically an associated rotation and drift. However, due to the varying longltudinal field at the solenoid ends, there must be an accompanying transverse magnetic fleld. This transverse field in fact results in a radial focusing term at the solenoid ends wnich is of course not present in the coordinate spinning arising from the torsion.

Finally, in Chapter 5 we describe the resulting Hamiltonian which has the form of a linearly coupled system. We review the parametrization of such a syster and discuss its stability.

\section{Hamiltonian Formulation}

In this chapter we will review the basic tools of Hamiltonian dynamics used in connection with planar accelerators. ${ }^{-4}$

\section{Specification of Coordinates}

A relativistic charged particle moving in a magnetic field is described by the Hamiltonian

$$
\vec{H}(\vec{p}, \vec{q}, t)=c\left[\left(\vec{p}-\frac{e}{c} \vec{A}\right)^{2}+m_{u}^{2} c^{2}\right]^{1 / 2}+e \Phi
$$

where $\Phi$ and $\AA$ are the scalar and vector potentials, and

$$
\vec{q}=(X, Y, Z), \quad \vec{p}=\left(p_{X}, P_{Y}, P_{Z}\right)
$$
momenta

We introduce a local coordinate system $\delta=(x, y, s)$ with canonical.

$$
\overrightarrow{\mathrm{P}}=\left(\mathrm{P}_{\mathrm{x}}, \mathrm{P}_{\mathrm{y}}, \mathrm{P}_{\mathrm{s}}\right)
$$

attached to a reference curve $\vec{r}_{0}(s)$ as shown in Fig. 1. The curve $\vec{r}_{0}(s)$ is defined so that it is a possible particle orbit, where 


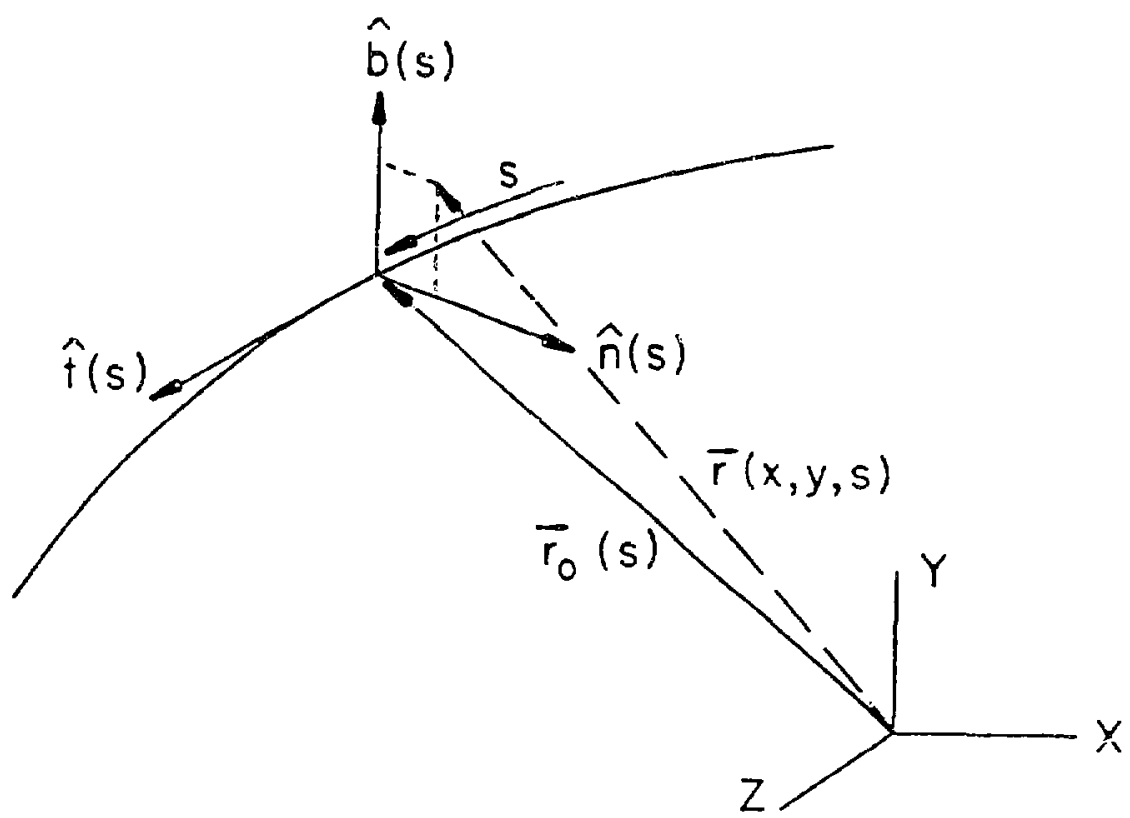

Fig. 1 .

$\vec{r}_{0}(s)$ is a reference curve

$\vec{r}(x, y, z)$ is any point in space

$\hat{i}(s)$ is the unit vector tangent to the reference curve

$\hat{\mathrm{n}}(\mathrm{s}) \quad$ is the principal normal vector

$\hat{b}(s)$ is the binormal unitary vector.

These vectors fulfill the following properties ${ }^{5}$

$$
\begin{gathered}
\frac{d \vec{r}_{Q}}{d s}=\hat{t} \quad ; \quad \frac{d \hat{t}}{d s}=\kappa \hat{n} \\
\frac{d \hat{n}}{d s}=\tau \hat{b}-k \hat{t} \quad ; \quad \frac{d \hat{b}}{d s}=-\tau \hat{n}
\end{gathered}
$$


where

$$
x=\left|\hat{t}^{\prime}\right| \quad, \tau=\left|\hat{b}^{\prime}\right|
$$

are the curvature and the torsion of the curve at $s$.

In this coordinate system, the Hamiltonian (2.1) takes the form

$$
\begin{gathered}
\vec{H}(\vec{P}, \vec{Q}, t)=c\left[\left(P_{x}-\frac{e}{c} A_{x}\right)^{2}+\left(P_{y}-\frac{e}{c} A_{y}\right)^{2}\right. \\
\left.+\left(\frac{\left(P_{s}-\frac{e}{c} A_{s}\right)-\tau x\left(P_{y}-\frac{e}{c} A_{y}\right)+\tau y\left(P_{x}-\frac{e}{c} A_{x}\right)}{1-k x}\right)^{2}+m_{o}^{2} c^{2}\right]^{1 / 2}+e \Phi
\end{gathered}
$$

where

$$
\begin{aligned}
& P_{x}=\vec{p} \cdot \hat{n}=p_{n} \\
& P_{y}=\vec{p} \cdot \hat{b}=p_{b} \\
& p_{s}=(1-k x) p_{t}+\tau\left(x p_{b}-y p_{n}\right)
\end{aligned}
$$

and the vector potential components $A_{x}, A_{y}$, $A_{s}$ fulfill similar relations.

In order to use the path length $s$ as independent parameter, we make the canonical transormation

$$
\begin{gathered}
s=t \\
-H=P_{s}=-\bar{H}
\end{gathered}
$$

where $H(\vec{P}, \vec{Q}, s)$ is the new Hamiltonian, given by

$$
\begin{aligned}
& H(\vec{P}, \vec{Q}, s)=-\frac{e}{c} A_{s}-\tau x\left(P_{y}-\frac{e}{c} A_{y}\right)+\tau y\left(P_{x}-\frac{e}{c} A_{x}\right) \\
& -(1-k x)\left[P_{s}^{2}-\left(P_{x}-\frac{e}{c} A_{x}\right)^{2}-\left(P_{y}-\frac{e}{c} A_{y}\right)^{2}\right] 1 / 2
\end{aligned}
$$


Here we have taken the case $\Phi=0$ so that

$$
(\mathrm{H} / \mathrm{c})^{2}-\mathrm{m}_{\mathrm{o}}^{2} \mathrm{c}^{2}=|\overrightarrow{\mathrm{P}}|^{2} \simeq \mathrm{P}_{\mathrm{s}}^{2}
$$

The equations of motion are obtalned from (2.9) through the Hamiltonian equations

$$
\begin{aligned}
& \mathrm{x}^{\prime}=\frac{\partial \mathrm{H}}{\partial \mathrm{P}_{\mathrm{x}}} \quad ; \quad \mathrm{y}^{\prime}=\frac{\partial \mathrm{H}}{\partial \mathrm{P}_{\mathrm{y}}} \\
& \mathrm{P}_{\mathrm{X}}^{\prime}=-\frac{\partial \mathrm{H}}{\partial \mathrm{x}} \quad ; \quad \mathrm{P}_{\mathrm{y}}^{\prime}=-\frac{\partial H}{\partial \mathrm{y}}
\end{aligned}
$$

where the derivative is now with respect to the parameter s.

\section{Motion with a Transverse Magnetic Field.}

\subsection{Determination of the Vector Potential}

In this section we are going to find the vector potential that gives the transverse dipole and quadrupole magnetic fleld components for the case in which the torsion $\tau \neq 0$. For $\tau=0$ we obtain the well known equations of motion.

$$
\begin{aligned}
& \text { We have, } \\
& \vec{r}_{G}=x_{0} \hat{i}+y_{0} \hat{j}+z_{0} \hat{k} \\
& \hat{n}=\alpha_{1} \hat{i}+\alpha_{2} \hat{j}+\alpha_{3} \hat{k} \\
& \hat{b}=\beta_{1} \hat{i}+\beta_{2} \hat{j}+\beta_{3} \hat{k} \\
& \hat{i}=\gamma_{1} \hat{i}+\gamma_{2} \hat{j}+\gamma_{3} \hat{k}
\end{aligned}
$$

and further,

$$
\begin{aligned}
& X=x_{0}(s)+\alpha_{1}(s) x+\beta_{1}(s) y \\
& Y=y_{0}(s)+\alpha_{2}(s) x+\beta_{3}(s) y \\
& Z=z_{0}(s)+\alpha_{3}(s) x+\beta_{3}(s) y
\end{aligned}
$$


Taking into account that

$$
\alpha_{i}^{\prime}=\tau \beta_{i}-k \gamma_{i}
$$

and

$$
\beta_{i}^{\prime}=-\tau \alpha_{i}, \quad \gamma_{i}^{\prime}=\kappa \alpha_{i}
$$

we have that

$$
\left[\begin{array}{l}
\partial / \partial x \\
\partial / \partial y \\
\partial / \partial s
\end{array}\right]=S\left[\begin{array}{l}
\partial / \partial X \\
\partial / \partial Y \\
\partial / \partial z
\end{array}\right]
$$

where

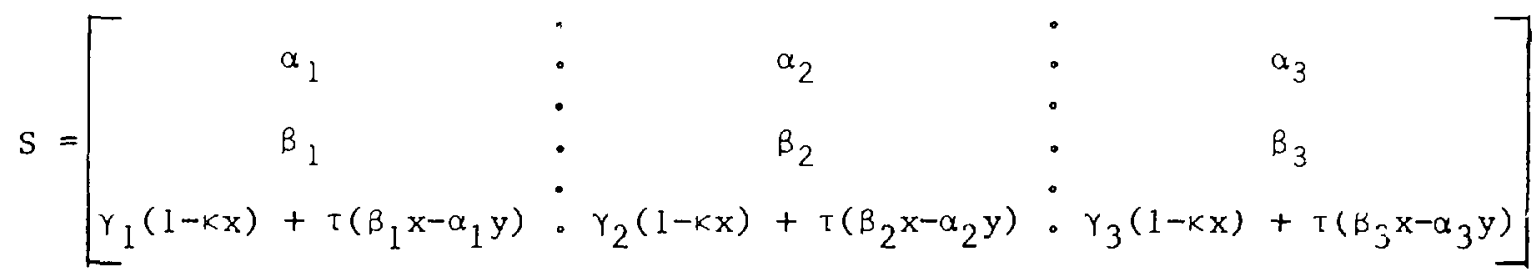

(3.4b)

Thus,

$$
\left[\begin{array}{l}
\partial / \partial X \\
\partial / \partial Y \\
\partial / \partial Z
\end{array}\right]=S^{-1}\left[\begin{array}{l}
\partial / \partial \mathrm{x} \\
\partial / \partial y \\
\partial / \partial S
\end{array}\right]
$$

where

$$
S^{-1}=\left[\begin{array}{ccc}
\alpha_{1}+\frac{\tau \gamma_{1} y}{1-\kappa x} & \beta_{1}-\frac{\tau \gamma_{1} x}{1-1 x} & \frac{\gamma_{1}}{1-\kappa x} \\
\alpha_{2}+\frac{\tau \gamma_{2} y}{1-\kappa x} & \beta_{2}-\frac{\tau \gamma_{2} x}{1-\kappa x} & \frac{\gamma_{2}}{1-\kappa x} \\
\alpha_{3}+\frac{\tau \gamma_{3} y}{1-\kappa x} & \beta_{3}-\frac{\tau \gamma_{3} x}{1-\kappa x} & \frac{\gamma_{3}}{1-\kappa x}
\end{array}\right]
$$


The momencum $\vec{P}$ and the vector potential $\dot{A}$ also will have the same transformation as $(3.4 a)$ and $(3.5 a)$, i.e.

$$
\left[\begin{array}{l}
\mathrm{P}_{\mathrm{X}} \\
\mathrm{P}_{\mathrm{Y}} \\
\mathrm{P}_{\mathrm{S}}
\end{array}\right]=\mathrm{s}\left[\begin{array}{l}
\mathrm{P}_{\mathrm{X}} \\
\mathrm{P}_{\mathrm{Y}} \\
\mathrm{P}_{\mathrm{Z}}
\end{array}\right] ;\left[\begin{array}{l}
\mathrm{A}_{\mathrm{X}} \\
\mathrm{A}_{\mathrm{Y}} \\
\mathrm{A}_{\mathrm{S}}
\end{array}\right]=\mathrm{s}\left[\begin{array}{l}
\mathrm{A}_{\mathrm{X}} \\
\mathrm{A}_{\mathrm{Y}} \\
\mathrm{A}_{\mathrm{Z}}
\end{array}\right]
$$

Calculating $\left(\vec{p}-\frac{e}{c} \AA\right)^{2}$ from eq. (3.6) we obtain the same expression that leads to the Hamiltonian (2.6).

Now we are interested in evaluating $\nabla \times A$ in the new coordinate system $(x, y, s)$. Using $(3.5 a),(3.6 b)$ and the inverse transformation of eq. (3.1),

$\nabla X A=\left|\begin{array}{ccc}\hat{i} & \hat{j} & \hat{k} \\ \partial / \partial X & \partial / \partial Y & \partial / \partial Z \\ A_{X} & A_{Y} & A_{Z}\end{array}\right|=$

$$
\begin{aligned}
& \alpha_{1} \hat{n}+\beta_{1} \hat{b}+\gamma_{1} \hat{t} \quad: \quad \alpha_{2} \hat{n}+\beta_{2} \hat{b}+\gamma_{2} \hat{t} \\
& \left(\alpha_{1}+\frac{\tau \gamma_{1} y}{1-\frac{K x}{x}}\right) \frac{\partial}{\partial x}+\left(\beta_{1}-\frac{\tau \gamma_{1} x}{1-\kappa x}\right) \frac{\partial}{\partial y}+\frac{\gamma_{1}}{1-K \gamma_{2}} \frac{\partial}{\partial s} \vdots\left(\alpha_{2}-\frac{\tau \gamma_{2} y}{1-K x}\right) \frac{\partial}{\partial x}+\left(\beta_{2}-\frac{\tau \gamma_{2} x}{1-K x}\right) \frac{\partial}{\partial y}+\frac{\gamma_{2}}{1-K x} \frac{\partial}{\partial s} \vdots \\
& \left(\alpha_{1}+\frac{\tau \gamma_{1} y}{1-\kappa x}\right) A_{x}+\left(\beta_{1}-\frac{\tau \gamma_{1} x}{1-\kappa x}\right) A_{y}+\frac{\gamma_{1}}{1-\kappa x} A s \vdots\left(\alpha_{2}+\frac{\tau \gamma_{2} y}{1-K x}\right) A_{x}+\left(\beta_{2}-\frac{\tau \gamma_{2} x}{1-K x}\right) A_{y}+\frac{T_{2}}{-K x} A_{S} \\
& \alpha_{3^{n}}+\beta_{3} \hat{b}+\gamma_{3} \hat{t} \\
& \left(\alpha_{3}+\frac{\tau \gamma_{3} y}{1-k x}\right) \frac{\partial}{\partial x}+\left(\beta_{3}-\frac{\tau \gamma_{3} x}{1-k x}\right) \frac{\partial}{\partial y}+\frac{\gamma_{3}}{1-k x} \frac{\partial}{\partial s} \\
& \left(\alpha_{3}+\frac{\tau \gamma_{3}{ }^{y}}{1}\right) A+\left(B-\frac{\tau \gamma_{3} x}{2}\right) A+\stackrel{\gamma_{3}}{ } A \\
& \begin{array}{lllllll}
1-K x & x & 3 & 1-K x & y & 1-K x & s
\end{array}
\end{aligned}
$$

$=\left\{\frac{\tau y}{I-K x}\left(\frac{\partial A_{x}}{\partial y}-\frac{\partial A_{y}}{\partial x}\right)+\frac{1}{I-K x}\left(\frac{\partial A_{s}}{\partial y}-\frac{\partial A_{y}}{\partial s}\right)\right\} \hat{n}$

$+\left\{\frac{\tau x}{I-K x}\left(\frac{\partial A_{y}}{\partial x}-\frac{\partial A x}{\partial y}\right)+\frac{1}{1-K x}\left(\frac{\partial A_{x}}{\partial s}-\frac{\partial A_{s}}{\partial x}\right)\right\} \hat{b}$

$+\left(\frac{\partial A}{\partial x} \Psi-\frac{\partial A}{\partial y}\right) \hat{t}$ 
In particular, if we only have a transverse field, that is,

$$
B_{t}=\frac{\partial A_{y}}{\partial x}-\frac{\partial A_{x}}{\partial y}=0
$$

then in the case of nonzero torsion in magnet elements, we have

$$
\begin{aligned}
& B_{n}=\frac{1}{I-K x}\left(\frac{\partial A_{s}}{\partial y}-\frac{\partial A_{y}}{\partial s}\right) \\
& B_{b}=\frac{1}{I-K x}\left(\frac{\partial A_{x}}{\partial s}-\frac{\partial A_{s}}{\partial x}\right)
\end{aligned}
$$

Now, although we restrict ourselves to zero torsion [ $\tau=0$ ] inside any magnecic elements, orbit torsion is froduced by having $\tau$ different from zero in field free regions. Thus, in this coordinate system, longitudinal fields are introduced in the "field free" regions. We find that the general expression (3.7) can be simplified and can be expressed as,

$\nabla x A=\frac{1}{1-K x}\left[\left(\frac{\partial A_{s}}{\partial y}-\frac{\partial A_{y}}{\partial s}\right) \hat{n}+\left(\frac{\partial A_{x}}{\partial s}-\frac{\partial A_{s}}{\partial x}\right) \hat{b}\right]+\left(\frac{\partial A_{y}}{\partial x}-\frac{\partial A_{x}}{\partial y}\right) \hat{t}$

We can choose, in eq. (3.8)

$$
A_{\mathrm{x}}=A_{\mathrm{y}}=0
$$

and we further assume the power series for $A_{t}$,

$$
A_{t}=a_{0}+a_{1} x+a_{2} y+a_{3} x^{2}+a_{4} x y+a_{5} y^{2}+\cdots \cdot
$$

Then, apriopriate magnet placement with respect to the defined reference orbit, together with the assumption of linear fields, lead to the magnetic field form,

$$
\begin{gathered}
B_{n}=B^{\prime} y \\
B_{b}=-B_{o}+B^{\prime} x
\end{gathered}
$$


which then leads to

$$
A_{S}=a_{0}+\left(a_{1}-x a_{0}\right) x+\left(a_{3}-k a_{1}\right) x^{2}+a_{5} y^{2}
$$

with

$$
\begin{gathered}
a_{1}-k a_{s}=B_{0} \\
-2 a_{3}+k a_{1}+k^{2} a_{0}=B^{\prime}
\end{gathered}
$$

To obtain a complete description of the vector potential we need a third condition, whlch is obtained by comparison with the well known equations of motion in torsion free regions. This is done in the next section.

\subsection{Equations of Motion}

$$
\begin{aligned}
& \text { Sirce } A_{x}=A_{y}=0, e q \cdot(2.9) \text { is reduced to } \\
& H=-\epsilon_{c}^{\epsilon} A_{s}+\tau\left(y P_{x}-x P_{y}\right)-(1-k x)\left[P_{s}^{2}-P_{x}^{2}-P_{y}^{2}\right]^{1 / 2}
\end{aligned}
$$

If we consider additionally a spread in the momentum, then for an cffmomentum particle we can express

$$
\mathrm{P}_{s}=\mathrm{P}_{\mathrm{o}}\left(1+\frac{\Delta \mathrm{P}}{\mathrm{P}}\right)
$$

and

$(1-k x)\left[P_{s}^{2}-P_{x}^{2}-P_{y}^{2}\right] \simeq P_{o}\left[1+(1-k x) \frac{\Delta P}{P}-k x-\frac{1}{2}\left(\frac{P_{x}}{P_{o}}\right)^{2}-\frac{1}{2}\left(\frac{P_{y}}{P_{o}}\right)^{2}\right]$

Using (3.13) and $(3.17)$ in (3.15)

$$
\begin{gathered}
H=-\frac{e}{c}\left(a_{0}+\left(a_{1}-k a_{0}\right) x+\left(a_{3}-k a_{1}\right) x^{2}+a_{5} y^{2}\right)+\tau\left(y P_{x}-x P_{y}\right) \\
-P_{0}\left[1+(1-k x) \frac{\Delta P}{P}-k x-\frac{1}{2}\left(\frac{P_{x}}{P_{0}}\right)^{2}-\frac{1}{2}\left(\frac{P_{y}}{P_{0}}\right)^{2}\right]
\end{gathered}
$$


The Hamiltonian equations are

$$
\begin{aligned}
& P_{x}^{\prime}=\frac{e}{c}\left(\left(a_{1}-\kappa a_{0}\right)+2\left(a_{3}-k a_{1}\right) x\right)+\tau F_{y}-P_{o} \kappa\left(\frac{A r}{P}+1\right) \\
& P_{y}^{\prime}=\frac{e}{c} B^{\prime} y-\tau P_{x} \\
& x^{\prime}=\frac{P_{x}}{P_{o}}+\tau y \\
& y^{\prime}=\frac{P_{y}}{P_{0}}-\tau x
\end{aligned}
$$

From (3.19) we get the equations

$$
x^{\prime \prime}-\left(\frac{2 e}{P_{0}}\left(a_{3}-k a_{1}\right)+\tau^{2}\right) x=\frac{e}{P_{0}}\left(a_{1}-k a_{0}\right)-k\left(\frac{\Delta P}{P}+1\right)+2 \tau y^{\prime}+\tau^{\prime} y^{\prime}
$$

and

$$
y^{\prime \prime}-\left(\frac{e}{P_{o}} B^{\prime}+\tau^{2}\right) h=-2 \tau x^{\prime}-\tau^{\prime} x
$$

For $\tau=0$ they must agree with

$$
\begin{aligned}
& x^{\prime \prime}+\left(k+k^{2}\right) x=-k \frac{\Delta P}{P} \\
& y^{\prime \prime}-k y=0
\end{aligned}
$$

where $K=\frac{B^{\prime}}{B_{0} \rho}$. Taking into account that $P_{0}=e B_{0} \rho$, the coefficients must satisfy

$$
a_{3}-k a_{1}=-\frac{1}{2}\left(B^{\prime}+K B_{0}\right)
$$

Solving (3.14) and (3.22) we get

$$
\begin{gathered}
a_{0}=0 \\
a_{1}=B_{0} \\
a_{3}=\frac{1}{2}\left(B_{0} k-B^{\prime}\right)
\end{gathered}
$$


and then eq. (3.13) is

$$
A_{s}=B_{o} x-\frac{1}{2}\left(B_{o} k+B^{\prime}\right) x^{2}+\frac{B^{\prime}}{2} y^{2}
$$

and the equations of motion (3.20) take the form

$$
\begin{aligned}
& x^{\prime \prime}+\left(k+\kappa^{2}-\tau^{2}\right) x=-\kappa \frac{\Delta P}{P}+2 \tau y^{\prime}+\tau^{\prime} y \\
& y^{\prime \prime}-\left(k+\tau^{2}\right) y=-2 \tau x^{\prime}-\tau^{\prime} x
\end{aligned}
$$

\section{Transfer Matrix for a Drift Space with Torsion}

We are now going to derive the equations of motion in our coordinate system for a straight segment with constant torsion $\tau$ and length $s$.

For this drift space we have

$$
K=0=K
$$

and then equations (3.25) are simply

$$
\begin{aligned}
& x^{\prime \prime}-\tau^{2} x=2 \tau y^{\prime}+\tau^{\prime} y \\
& y^{\prime \prime}-\tau^{2} y=-2 \tau x^{\prime}-\tau^{\prime} x
\end{aligned}
$$

We can easily see that the solution of equations $(4.2)$ are

$$
\begin{aligned}
& x(s)=(a+b s) \cos \theta+(c+d s) \sin \theta \\
& y(s)=(c+d s) \cos \theta-(a+b s) \sin \theta
\end{aligned}
$$

where $a, b, c, d$ are constants, and

$$
\frac{d \theta}{d s}=\tau(s)
$$


We can see from the appendix that having torsion in the straight sections is a direct consequence, having bending dipoles in intersecting planes. We will show that the transfer matrix is independent of $\tau$ explicitly. Thus, we can choose the length over which $\tau \neq 0$ arbitrarily. In particular we can choose $\tau=0$ in all elements (quadrupoles) between $d 1-$ noles. Also, since the transfer matrix is independent of $\tau$ explicitly, we can replace the transfer matrix by the prodict of a drift followed by á zero drift rotation.

Our assumptions about the reference orbit and magnet placement imply that $\kappa(s)$ and $\tau(s)$ are step functions. So, we musi keep in mind that there are $\delta$-function contributions to thelr derivatives.

In what follows we will study in detail the case $\tau:(s)=$ unstant $\rightarrow \infty$, in two situations:

a) when the transfer matrix relates to the canonical phar space,

b) when the transfer matrix relates to the $\left(x, x^{\prime}, y^{\prime}, y^{\prime}\right)$ space.

Let us assume that $\tau(s)$ is of the form

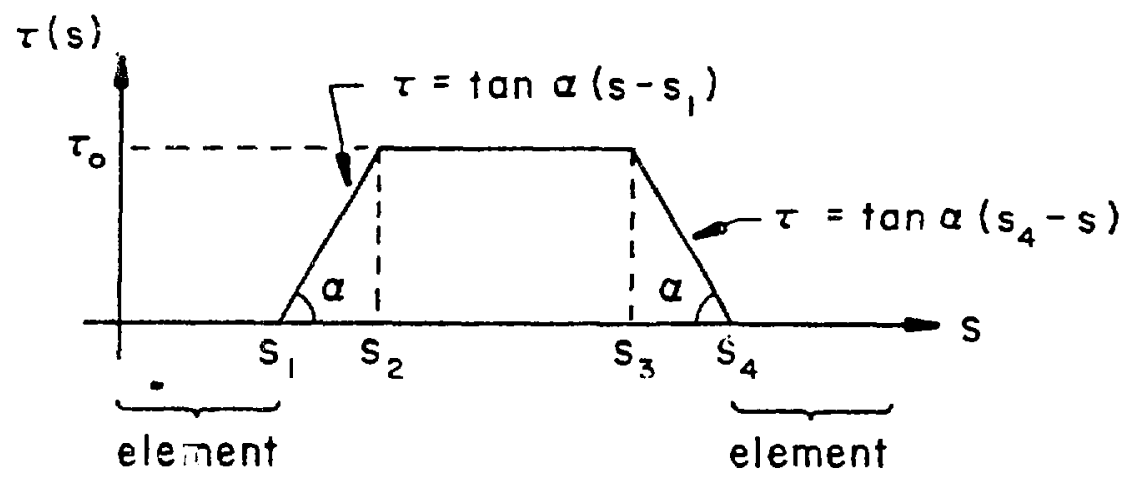

and $x_{1}, x_{1}^{\prime}, P_{x 1}, y_{1}, y_{1}^{\prime}, P_{v 1}$ be initial values at $s_{1}$ 


\section{Case a.}

First we will find the transfer matrix for the region where $\tau=\tan \alpha\left(s-s_{1}\right)$. Putting initial conditions (4.4) in eqs. (4.3), we have

$$
\left[\begin{array}{l}
\mathrm{x} \\
\mathrm{P}_{\mathrm{x}} \\
\mathrm{y} \\
\mathrm{P}_{\mathrm{y}}
\end{array}\right]=\left[\begin{array}{cc|cc}
\cos \theta_{2} & -G\left(s-s_{1}\right) \cos \theta_{2} & \sin \theta_{2} & -G\left(s-s_{1}\right) \sin \theta_{2} \\
0 & \cos \theta_{2} & \mathrm{sin} \theta_{2} \\
-\sin \theta_{2} & G\left(s-s_{1}\right) \sin \theta_{2} & \cos \theta_{2} & -G\left(s-s_{1}\right) \cos \theta_{2} \\
0 & -\sin \theta_{2} & 0 & \cos \theta_{2}
\end{array}\right]\left[\begin{array}{l}
x_{1} \\
P_{x_{1}} \\
y_{1} \\
P_{y 1}
\end{array}\right]
$$

where

$$
\theta_{2}=\frac{\tau_{0}}{2} \frac{\left(s-s_{1}\right)^{z}}{s_{2}-s_{1}}
$$

If we now take $s=s_{2}$ and take the 1 imit $s_{2} \rightarrow s_{1}$

$$
\left[\begin{array}{c}
\mathrm{x}_{2} \\
\mathrm{P}_{\mathrm{x} 2} \\
\mathrm{y}_{2}
\end{array}\right]=\mathrm{I}\left[\begin{array}{c}
\mathrm{x}_{1} \\
\mathrm{P}_{\mathrm{x} 1} \\
\mathrm{y}_{1} \\
\mathrm{P}_{\mathrm{y} 1}
\end{array}\right]
$$

Since $I^{-1}=I$, we expect that in the region where $\tau=$ ian $\alpha\left(s_{4}-s\right)$ the transfer matrix will be again I, and therefore, ir going from $s_{1}$ to $s_{4}$, the only significant matrix in the play is in the region where $\tau=\tau_{0}$, which now calcuat's. 
Using (4.3) amd (4.6), we have

$\left[\begin{array}{l}\mathrm{x} \\ \mathrm{P}_{\mathrm{x}} \\ \mathrm{y} \\ \mathrm{P}_{\mathrm{y}}\end{array}\right]=\left[\begin{array}{rrr|rrr}\cos \theta_{3} & G\left(s-s_{2}\right) & \cos \theta_{3} & \sin \theta_{3} & G\left(s-s_{2}\right) & \sin \theta_{3} \\ 0 & & \sin \theta_{3} & 0 & \sin \theta_{3} \\ -\sin \theta_{3} & -G\left(s-s_{2}\right) & \sin \theta_{3} & \cos \theta_{3} & G\left(s-s_{2}\right) & \cos \theta_{3} \\ 0 & & -\sin \theta_{3} & & & \cos \theta_{3}\end{array}\right]\left[\begin{array}{l}x_{2} \\ P_{x 2} \\ y \\ P_{y 2}\end{array}\right]$

$(4.7 a)$

where

$$
\theta_{3}=\tau_{0}\left(s-s_{2}\right) . \quad G=\frac{1}{P_{0}}
$$
write

If in (4.7a) we make $s=s_{3}$ and $\ell=s_{3} s_{2}$ and $\bar{\theta}_{3}=\tau_{0}\left(s_{3}-s_{2}\right)$, we can

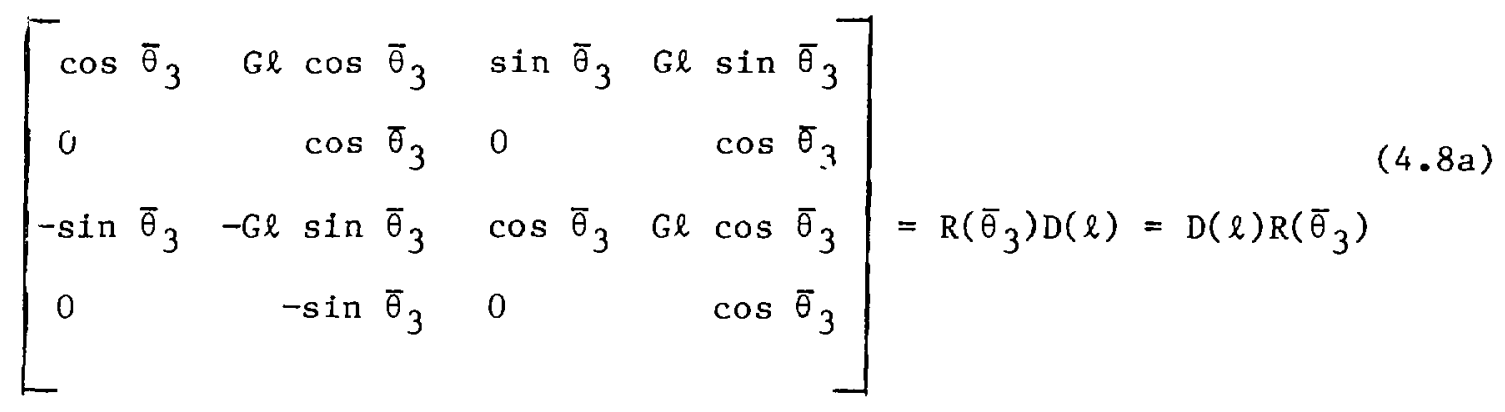

where

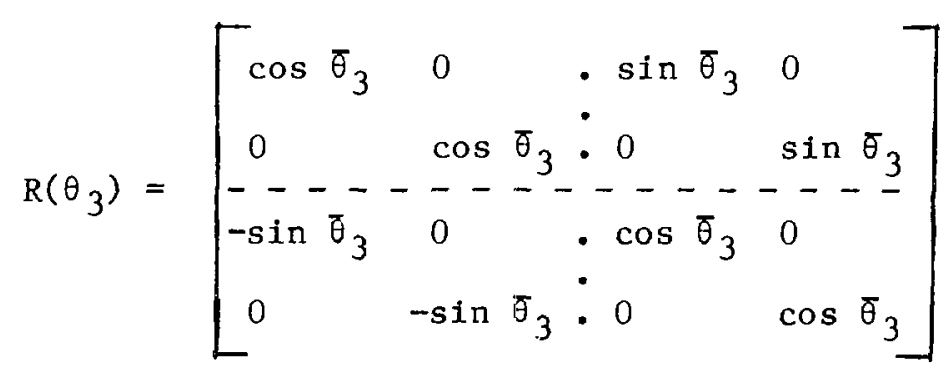


is a rotation in each phase space, and

$$
D(\ell)=\left[\begin{array}{llll}
1 & G l & 0 & 0 \\
0 & 1 & 0 & 0 \\
0 & 0 & 1 & G l \\
0 & 0 & 0 & 1
\end{array}\right]
$$

is the matrix corresponding to a normal drift space.

\section{Case b.}

For this case, the transfer matrix for going frow $s_{1}$ to $s$, for $s_{1} \leqslant s \leqslant s_{2}$ is,

\begin{tabular}{|c|c|c|c|}
\hline$x$ & $\cos \theta$ & $-\left(s-s_{l}\right) \cos \theta$ & - $\begin{array}{ccc} & \sin \theta & -\left(s_{-}-s_{1}\right) \sin \theta \\
2 & 2 & 2\end{array}$ \\
\hline$x^{\prime}$ & $\begin{array}{l}-\tau \sin \theta_{2} \\
=\sin { }^{-}{ }_{2}^{-}\end{array}$ & $\begin{array}{l}-\left(s-s_{1}\right) \tau \sin \theta_{2}+\cos \theta_{2} \\
-\left(\bar{s}_{1} \bar{s}_{1}\right) \sin \bar{\theta}_{2}^{2}---{ }^{2}-\end{array}$ & $\begin{array}{c}\tau \cos \theta_{2} \sin \theta_{2}+\left(s-s_{1}\right) \tau \cos \theta \\
:-\overline{c o s} \theta_{2}-{ }^{2}=\left(\bar{s}-\bar{s}_{1}^{2}\right) \overline{c o s}^{1} \bar{\theta}_{2}-{ }^{2}\end{array}$ \\
\hline$y^{\prime}$ & $-\tau \cos \theta_{2}$ & $-\sin \theta_{2}-\left(s-s_{1}\right) \tau \cos \theta_{2}$ & $\cos \theta_{2}-\left(s-s_{1}\right) \tau \sin \theta_{2}$ \\
\hline
\end{tabular}

Where $\theta_{2}$ is given by eq. (4.5b).

Taking $\mathrm{s}^{=} \mathrm{s}_{2}$ and $\mathrm{s}_{2} \rightarrow \mathrm{s}_{1}$,

$$
\underset{s \rightarrow s}{\lim [\operatorname{matrix}(4.9)]}\left[\begin{array}{cccc}
1 & 0 & 0 & 0 \\
0 & 1 & \tau & 0 \\
0 & 0 & 1 & 0 \\
\tau & 0 & 0 & 1 \\
0 & & &
\end{array}\right]
$$


Direct calcujations show that the matrix for going from $s_{2}$ to $s_{3}$ is

$$
\left[\begin{array}{crrrr}
\cos \bar{\theta}_{3}+\bar{\theta}_{3} \sin \bar{\theta}_{3} & \left(s_{3}-s_{2}\right) \cos \bar{\theta}_{3} & \sin \bar{\theta}_{3}-\bar{\theta}_{3} \cos \bar{\theta}_{3} & \left(s_{3}-s_{2}\right) \sin \bar{\theta}_{3} \\
\tau_{0} \bar{\theta}_{3} \cos \bar{\theta}_{3} & \cos \bar{\theta}_{3}-\bar{\theta}_{3} \sin \bar{\theta}_{3} & \tau_{0} \bar{\theta}_{3} \sin \bar{\theta}_{3} & \sin \bar{\theta}_{3}+\bar{\theta}_{3} \cos \bar{\theta}_{3} \\
-\sin \bar{\theta}_{3}+\bar{\theta}_{3} \cos \bar{\theta}_{3} & -\left(s_{3}-s_{2}\right) \sin \bar{\theta}_{3} & \cos \bar{\theta}_{3}+\bar{\theta}_{3} \sin \bar{\theta}_{3} & \left(\operatorname{s}_{3}-s_{2}\right) \cos \bar{\theta}_{3} \\
-\tau_{0} \bar{\theta}_{3} \sin \bar{\theta}_{3} & -\sin \theta_{3}-\bar{\theta}_{3} \cos \bar{\theta}_{3} & \tau_{0} \bar{\theta}_{3} \cos \bar{\theta}_{3} & \cos \bar{\theta}_{3}-\bar{\theta}_{3} \sin \bar{\theta}_{3}
\end{array}\right]
$$

For the section between $s_{3}$ and $s_{4}$, the watrix must be the inverse of (i. 10), i.e.,

$$
\left[\begin{array}{cccc}
1 & 0 & 0 & 0 \\
0 & 1 & -\tau & 0 \\
0 & 0 & 1 & 0 \\
\tau_{0} & 0 & 0 & 1
\end{array}\right]
$$

Thus, the transfer matrix from $s$ to $s_{4}$ is the product of $(4.10),(4.11)$ and (4.12). Performing the prodult, we obtain

$$
\left[\begin{array}{cccc}
\cos \bar{\theta}_{3} & \left(s_{3}-s_{2}\right) \cos \bar{\theta}_{3} & \sin \bar{\theta}_{3} & \left(s_{3}-s_{2}\right) \sin \bar{\theta}_{3} \\
0 & \cos \bar{\theta}_{3} & 0 & \sin \bar{\theta}_{3} \\
-\sin \bar{\theta}_{3} & -\left(s_{3}-s_{2}\right) \sin \bar{\theta}_{3} & \cos \bar{\theta}_{3} & \left(s_{3}-s_{2}\right) \cos \bar{\theta}_{3} \\
0 & -\sin \bar{\theta}_{3} & 0 & \cos \theta_{3}
\end{array}\right]
$$

which is similar to $(4,7 a)$, with $G=1$, and therefore can be factorized as $(4.8 a)$, i.e., it is the product of a rotation matrix times a drift space. 
Thus, we have proved that the transfer matrix depends only on the coordinate rotation angle and not on $\tau$ explicity and also that the observed physics in regions of non-zero $\tau$ depend on the rate of the spinning coordinate system, $\tau$.

\section{Analytic Treatment of Equations of Motion}

Using eq. (3.13) we can express the Hamiltonian (3.15) as

$$
H=\frac{e B^{\prime}}{2 c}\left(x^{2}-y^{2}\right)+\tau\left(y P_{x}-x P_{y}\right)+\frac{1}{2} \frac{P_{x}^{2}}{P_{o}}+\frac{1}{2} \frac{P_{y}^{2}}{P_{o}} \quad \text { (5.1a) }
$$

where we make $B_{0}=0, k=0, \Delta P, P=0$

and we fgnore the constant 1 .

Hamfltontans of the type (5.1a) have already been studied ${ }^{6}$, and hence, we will only give the solution for our casa.

We define $\mathrm{X}$ as

$$
\mathrm{X} \equiv\left[\begin{array}{l}
\mathrm{x} \\
\mathrm{P}_{\mathrm{x}} \\
\mathrm{P}_{\mathrm{y}}
\end{array}\right]
$$

A similar analysis to ref. 6 shows that $X$ can be expressed as

$$
\mathrm{X}=\mathrm{RV}
$$

where

$$
R=\left[\begin{array}{lll}
I & \cos \phi & D^{-1} \sin \phi \\
-D \sin \phi & I \cos \phi
\end{array}\right]
$$


and

$$
v \equiv\left[\begin{array}{l}
u \\
P_{u} \\
v \\
P_{u}
\end{array}\right]=\left[\begin{array}{l}
\left(w_{1} \beta_{1}\right)^{1 / 2} \cos \psi_{1} \\
-\left(w_{1} / \beta_{1}\right)^{1 / 2}\left(\sin \psi_{1}+\alpha_{1} \cos \psi_{1}\right) \\
\left(w_{2} \beta_{2}\right)^{1 / 2} \cos \psi_{2} \\
-\left(w_{2} / \beta_{2}\right)^{1 / 2}\left(\sin \psi_{2}+\alpha_{2} \cos \psi_{2}\right)
\end{array}\right]
$$

In eq. (5.3) $\phi$ and the 3 indepeadent parameters $0^{+}$

$$
D=\left[\begin{array}{ll}
a & b \\
c & d
\end{array}\right] \quad ; \quad(a d-b c=1)
$$

are parameters of the group $S p(4)$ and fulfill the conditions:

$$
\begin{aligned}
& \phi^{\prime}=\frac{1}{2} \tau(a+d) \\
& a^{\prime}=b F+c Z+\tau(2-a(a+d)) \cot 2 \phi \\
& b^{\prime}=-(a-d) Z-\tau b(a+d) \cot 2 \phi \\
& c^{\prime}=(a+d) F \cdots \tau c(a+d) \cot 2 \phi \\
& d^{\prime}=b F-c Z+\tau(2-d(a+d)) \cot 2 \phi
\end{aligned}
$$

where

$$
\mathrm{F}=\frac{\mathrm{eB}^{\prime}}{\mathrm{c}} \quad \text { and } \quad \mathrm{Z}=\frac{1}{\mathrm{P}_{\mathrm{O}}}
$$

In eq. (5.4) there apfear the extended Courant-Snyder ${ }^{1}$ parameters which here satisfy the conditions

$$
\begin{aligned}
& \alpha_{1}^{\prime}=-Z Y_{1}+F \beta_{1}+\tau\left(b Y_{1}+c \beta_{1}\right) \tan \phi \\
& \beta_{i}^{\prime}=-2 Z \alpha_{1}+\tau\left(2 b \alpha_{1}-(a-d) \beta_{1}\right) \tan \phi \\
& \gamma_{1}^{\prime}=2 F \alpha_{1}+\tau\left(2 c \alpha_{1}+(a-d) \gamma_{1}\right) \tan \phi
\end{aligned}
$$




$$
\begin{aligned}
& \alpha_{2}^{\prime}=-Z \gamma_{2}-F \beta_{2}-\tau\left(b \gamma_{2}+c \beta_{2}\right) \tan \phi \\
& \beta_{2}^{\prime}=-2 Z \alpha_{2}-\tau\left(2 b \alpha_{2}-(a-d) \beta_{2}\right) \tan \phi \\
& \gamma_{2}^{\prime}=-2 F \alpha_{2}-\tau\left(2 c \alpha_{2}+(a-d) \gamma_{2}\right) \tan \phi \\
& \psi_{1}=\int \frac{(z-\tau b \tan \phi)}{\beta} d s-\delta_{1} \\
& \psi_{2}=\int \frac{(Z+\tau b \tan \phi)}{\beta} d s-\delta_{2}
\end{aligned}
$$

$W_{1}, W_{2}$ in $(5.4)$ and $\delta_{1}, \delta_{2}$ in (5.7) aro the constants of integration. $W_{1}$ and $W_{2}$ are given by

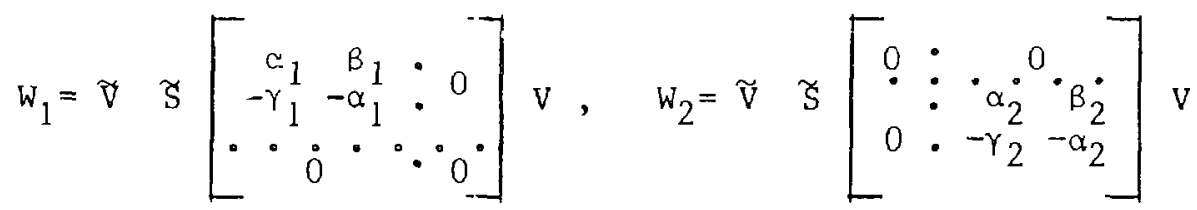

\section{Stability of Motion}

The transfer matrix for one turn, can be expressed as ${ }^{6}$

$$
\mathrm{m}=\text { RUR-1 }
$$

where $R$ is given by $(5.3)$ ana $U$ is

$$
\mathrm{U}=\left(\begin{array}{ll}
\mathrm{A} & 0 \\
0 & \mathrm{~B}
\end{array}\right)
$$

The stability of the motion can be studied for each normal coordinate separately, since the motion is decoupled in this system. Note that although the motion is simply harmonic in the normal coordinate system, when viewed in the real frame of reference $\left(x, x^{\prime}, y, y^{\prime}\right)$, this simplicity becomes hidden in coupled 4D phase space motion. The matrices, $A, B$, are the $2 \times 2$ symplectic matrices parametrized by 


$$
\begin{aligned}
& A=I \cos \mu_{1}+\left(\begin{array}{cc}
\alpha_{1} & \beta_{1} \\
-\gamma_{1} & -\alpha_{1}
\end{array}\right) \sin \mu_{1} \\
& B=I \cos \mu_{2}+\left(\begin{array}{rr}
\alpha_{2} & \beta_{2} \\
-\gamma_{2} & -\alpha_{2}
\end{array}\right) \text { sin } \mu_{2}
\end{aligned}
$$

For $n$ revolutions the transfer matrix is

$$
\mathrm{T}^{\mathrm{n}}=\mathrm{RU}^{\mathrm{n}} \mathrm{R}^{-1}=\mathrm{R}\left(\begin{array}{ll}
\mathrm{A}^{\mathrm{n}} & 0 \\
0 & \mathrm{~B}^{\mathrm{n}}
\end{array}\right) \mathrm{R}^{-1}
$$

But

$$
\mathrm{A}^{\mathrm{n}}=\mathrm{I} \cos \mathrm{n} \mu_{1}+\left(\begin{array}{cc}
\alpha_{1} & \beta_{1} \\
-\gamma_{1} & -\alpha_{1}
\end{array}\right) \sin \mathrm{n} \mu_{1}
$$

and there is similar relation for $B^{n}$. So the motion will be stable if $\mu_{1}, \mu_{2}$ are real, i.e., if

$$
|\operatorname{TrA}| \leqslant 2 \text { and }|\operatorname{TrB}| \leqslant 2 \text {. }
$$




\section{Appendix A}

In this appendix we analyze the torsion for a drift space between dipoles, as is shown in the next figure.

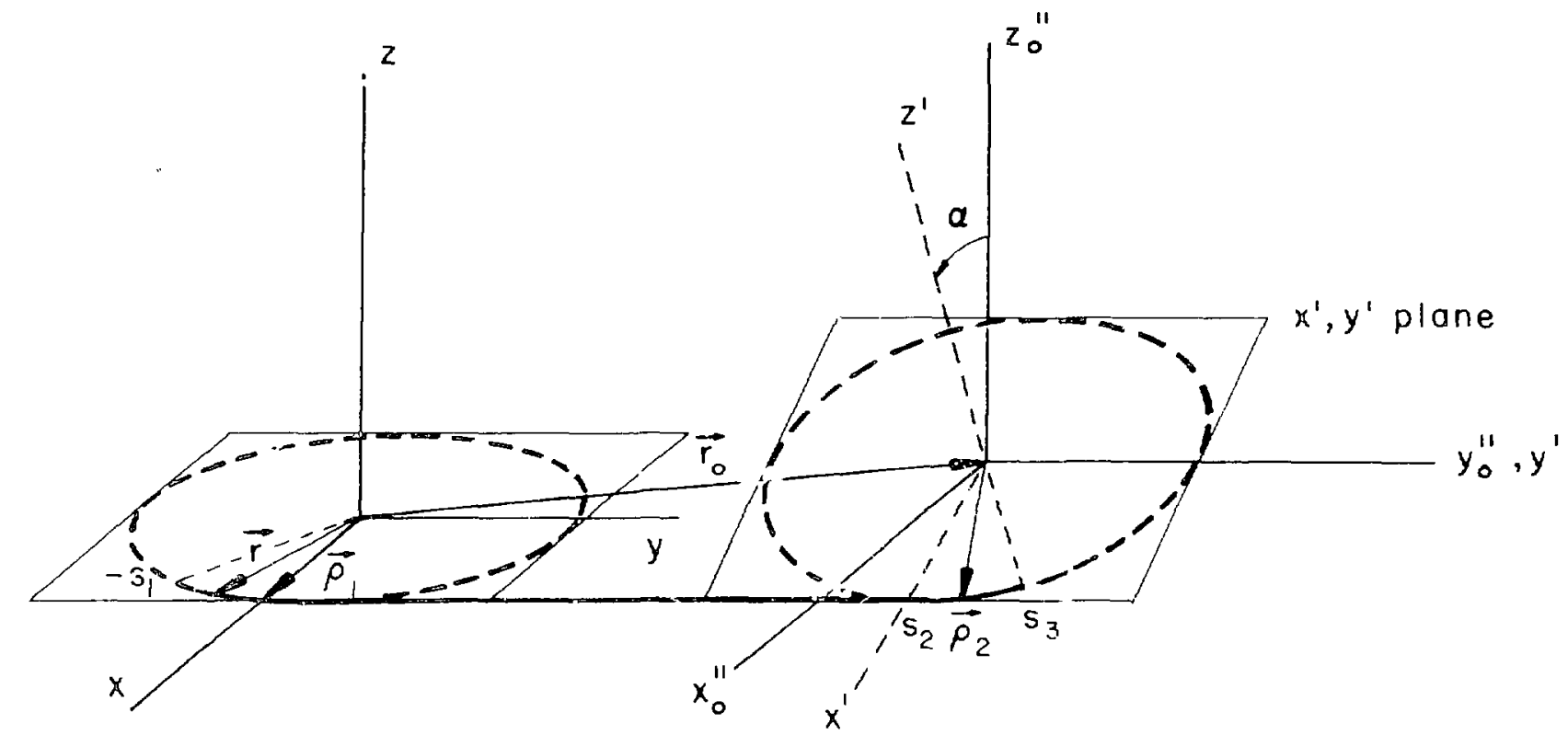

where

$$
\begin{aligned}
& \vec{r}_{0}=x_{0} \hat{i}+y_{0} \hat{j}+z_{0} \hat{k} \\
& \hat{i}^{\prime}=\cos \alpha \hat{i}-\sin \alpha \hat{k} \\
& \hat{j}^{\prime}=\hat{j}
\end{aligned}
$$

For the left circle

$$
\vec{r}(s)=\rho_{1} \cos \frac{s}{\rho_{1}} \hat{i}+\rho_{1} \sin \frac{s}{\rho_{1}} \hat{j}
$$

and

$$
\overrightarrow{\mathbf{r}}(0)=\vec{p}_{1}=\rho_{1} \hat{\dot{i}}
$$


and

$$
\begin{aligned}
& \hat{t}=\frac{d \vec{r}}{d s}=-\sin \frac{s}{\rho_{1}} \hat{i}+\cos \frac{s}{\rho_{1}} \hat{j} \\
& \hat{t}_{1} \equiv \hat{t}(o)=\hat{j}
\end{aligned}
$$

For the right circle

$$
\begin{aligned}
\vec{\rho}_{2}(s)=x^{\prime} \hat{i}+y^{\prime} \hat{k} & =\rho_{2} \cos \frac{s-s_{2}}{\rho_{2}} \cos \alpha \hat{j}+\rho_{2} \sin \frac{s-s_{2}}{\rho_{2}} \hat{j} \\
& -\rho_{2} \cos \frac{s-s_{2}}{\rho_{2}} \text { sin } \alpha \hat{k}
\end{aligned}
$$

We can express the position as

$$
\vec{r}(s)= \begin{cases}\rho_{1} \cos \frac{s}{\rho_{1}} \hat{i}+\rho_{1} \sin \frac{s}{\rho_{i}} \grave{j} & -s_{1} \leqslant 0 \\ \vec{\rho}_{1}+s \hat{t}_{1} & 0<s<s_{2} \\ \vec{r}_{0}+\vec{\rho}_{2} & s_{2} \leqslant s \leqslant s_{3}\end{cases}
$$

Using (A.1), (A.2) and (A.3) into (A.4) and asking for $\vec{r}(\mathrm{~s})$ to be a continuous curve, we get

$$
\begin{gathered}
x_{0}=\rho_{1}-\rho_{2} \cos \alpha \\
y_{0}=s_{2} \\
z_{0}=\rho_{2} \sin \alpha
\end{gathered}
$$

The tangent vector is

$$
E(s)=\left\{\begin{array}{cc}
-\sin \frac{s}{\rho_{1}} \hat{i}+\cos \frac{s}{\rho_{1}} \hat{j} & -s_{1} \leqslant 0 \\
\hat{j} & 0<s<s_{2} \\
-\sin \frac{s-s_{2}}{\rho_{2}} \cos \alpha \hat{i}+\cos \frac{s-s_{2}}{\rho_{2}} \hat{j}+\sin \frac{s-s_{2}}{\rho_{2}} \sin \alpha \hat{k} s_{2} s s_{3} s_{3}
\end{array}\right.
$$


and its derivative is

$$
\frac{d \hat{t}}{d s}= \begin{cases}\frac{1}{\rho_{1}}\left(-\cos \frac{s}{\rho_{1}} \hat{i}-\sin \frac{s}{\rho_{1}} \hat{j}\right)=k_{1} \hat{n}_{1} & -s \leqslant 0 \\ v(\cos \beta \hat{i}-\sin \beta \hat{k})=\kappa_{0} \hat{n}_{0} & 0<s<s_{0} \\ \frac{1}{\rho_{2}}\left(-\cos \frac{s-s_{2}}{\rho_{2}} \cos \alpha \hat{i}-\sin \frac{s-s_{2}}{\rho_{2}} \hat{j}+\cos \frac{s-s_{2}}{\rho_{2}} \sin \alpha \hat{k}\right)=k_{2} \hat{n}_{2} & s_{2} \leqslant s_{3} \leqslant\end{cases}
$$

The binormal vector $\hat{b}=\hat{t} \times \hat{n}$ is

$b(s)=\left\{\begin{array}{cc}\hat{k} & -s_{1} \leqslant 0 \\ -\sin \beta \hat{i}-\cos \beta \hat{k} & 0<s<s_{2} \\ \sin \alpha \hat{i}+\cos \alpha \hat{k} & s_{2} \leqslant s \leqslant s_{3}\end{array}\right.$

and its derivative is

$\frac{\hat{d u}}{d s}=\left\{\begin{array}{l}0 \\ -\beta^{\prime}(\cos \beta \hat{i}-\sin \beta \hat{k})=-\tau_{0} \hat{n}_{0}\end{array}\right.$

and then

$$
B^{\prime}=\tau_{0} \text { or } B(s)=\tau_{0} s+c
$$

Consequent ly

and

$$
\cos 2=A \cos \tau_{0} s-B \sin \tau_{0} s
$$

$$
\sin \beta=A \sin \tau_{0} s+B \cos \tau_{0} s
$$

where

$$
\mathrm{A}=\cos \mathrm{C}, \mathrm{B}=\sin \mathrm{C}
$$


From eqs. (A.7) we see that continuity of $\hat{n}$ implies that at $s=0$

$$
\begin{aligned}
& \cos \beta(0)=-1 \\
& \sin \beta(0)=0
\end{aligned}
$$

and

$$
\text { at } \begin{aligned}
s=s_{2}, & \cos \beta\left(s_{2}\right)=-\cos \alpha \\
& \sin \beta\left(s_{2}\right)=\sin \alpha
\end{aligned}
$$

Equations (A.10) and $(A .11)$ give

$$
\Lambda=-1 \quad, \mathrm{~B}=0
$$

and (A.10) with condition (A.13) and (A.12) give

$$
\begin{aligned}
& \cos \tau_{0} s_{2}=\cos \alpha \\
& \sin \tau_{0} s_{2}=\sin \alpha
\end{aligned}
$$

from which we find the torsion

$$
\tau_{0}=\frac{\alpha}{s_{2}}
$$

and thus

$$
\hat{b}(s)= \begin{cases}\hat{k} & -s_{1} \leqslant 0 \\ \sin \tau_{0} s \hat{i}+\cos \tau_{0} s \hat{k} & 0<s<s_{2} \\ \hat{k}^{\prime} & s_{2} \leqslant s \leqslant s_{3}\end{cases}
$$




\section{$\underline{\text { References }}$}

1. E.D Courant and S. Snyder, Ann. Phys, 3, 1 (1958).

2. H. Bruck, Accélérateurs Circulaires de Particlules (Presses Universitaires de France, Paris, 1966).

3. G. Guignard, A General Treatment of Resonances in Accelerators, CERN 78-11 (1978).

4. B.W. Montague, Single Particle Dynamics-Hamiltonian Formulation CERN 77-13 (1977).

5. M. Schwartz, S. Green and W.A. Rutledge, Vector Analysis (Harper \& Brothers), 1960.

6. D.A. Edwards and L.C. Teng, Parametrization of Linear Coupled Motion. FNAL; IEEE Trans. Nuc1. Sci. NS-20, No. 3, 885 (1973). 